\title{
AN ENSEMBLE BASED SYSTEM FOR CHLOROPHYLL-A ESTIMATION USING MODIS IMAGERY OVER SOUTHERN QUEBEC INLAND WATERS
}

\author{
Anas El-Alem ${ }^{1,{ }^{*}}$ Karem Chokmani ${ }^{1}$, Isabelle Laurion ${ }^{1}$ and Sallah E. El-Adlouni ${ }^{2}$ \\ 1 Institut National de la Recherche Scientifique Centre Eau-Terre-Environnement \\ 2 Moncton University
}

\begin{abstract}
The purpose of this study was to enhance the performance of the Adaptive-model (AM) for Chlorophyll-a (Chl-a) concentration estimation using MODIS-imagery. The AM is based on the combination of spectral response classification and three semi-empirical estimators. Most critical step in Chl-a estimation when using the AM is the estimator selection. A wrong selection could lead to an over- or underestimation and induces a staircase effect in Chl-a modelization. Ensemble based systems (EBS) development can be in interesting solution. One of the most used techniques to develop an EBS is bagging-algorithm. However, its main weakness is time consumption. The Gaussian-quadrature (GQ) formula has the potential to handle this kind of problems. The objective of this study is to develop a mixed ensemble based system for Chl-a estimation in Quebec inland waters using GQ formula. Statistical indexes evaluation was satisfied, relativeRMSE $=15 \%$ and $\mathrm{R}^{2}=0.98$ whereas matrix-confusion results were 0.92 and 0.8 for global-success and Kappa-index, respectively.
\end{abstract}

Index Terms - MODIS, HAB, Chl-a, Ensemble based systems, Gaussian quadrature.

\section{INTRODUCTION}

In reason of industrial, agricultural, and socio-economic development, the quality of many lakes has decreased because of the installation of Harmful algal blooms (HAB). On the other hand, with computer tools and satellite sensors development, remotely sensed data are increasingly used for monitoring $\mathrm{HAB}$ in inland waters [1]. HAB monitoring is possible due to the bio-optical activity of their principal pigment, the Chlorophyll-a (Chl-a), through bio-optical models linking inherent and apparent optical properties of water bodies. Thus, several models [2], indexes [3], and approaches [4] designed to estimate Chl-a concentrations in turbid water were recently developed, especially using medium-resolution spectroradiometers such as MODIS and MERIS sensors.

In this context, an Adaptive-model (AM) to estimate Chl-a concentration inland water bodies was recently developed based on the combination of water spectral response classification and three semi-empirical estimators. Discrimination between three trophic levels (waters poorly, moderately, and highly loaded in Chl-a), using a classifier, is firstly made before estimating the Chl-a with the corresponding estimator to the pre-identified trophic level (Figure 1 [5]). The classifier calibration was made using CART algorithm on the training data water spectral response. Once the AM classifier was calibrated, it was possible to sub-divide the same training data into three subgroups allowing to calibrate three specific estimators.



Figure 1. Simplified scheme of the intern structure of the AM. $\mathrm{V}_{1^{-}}$ Threshold and $\mathrm{V}_{2}$-Threshold are the thresholds calculated by CART using, respectively, the Variable- 1 and the Variable- 2 on the training data.

Besides, most critical step in estimating Chl-a using the AM is the estimator selection. Indeed, Decision trees algorithms are known by their instability [6]. A wrong selection of a given estimator could then lead to a significant over- or under-estimation and induces a staircase effect to Chl-a estimations. Ensemble based classifiers (EBC) development seems to well manage this kind of classification problems [7]. On the other hand, because of modeling Chl-a concentration complexity in inland water bodies, many authors [8] suggest to seek for several estimators outputs 
before making a decision. The combination of all this outputs appears to be the most informative one. This technique, known under the name of an Ensemble based estimators (EBE), is highly used in hydrological [9] and financial [10] studies.

Among main keys to develop a strong EBC or EBE called also, in a general way, Ensemble based systems (BES) is to reach the highest diversity between the BES elements (classifiers or estimators [11]). One of the earliest, intuitive and, the simplest methods of an EBS development is the Breiman's bagging; short for bootstrap aggregating [12]. Diversity in bagging is obtained by using bootstrapped resampling on the training data; this allows covering almost all solution space. However, main weakness of this method is time consumption, particularly in imagery processing, due to the high number of both the EBS elements and images pixels.

The Gaussian-quadrature (GQ) formula could be an interesting method to solve this problematic. This method, frequently used in uncertainty propagation analysis [13], has the potential to convert re-sampling vector problem, which requires arduous calculations, to an integration problem where the numerical resolution techniques are simpler and are accurate and approved [14]. This technique is very suited for practical applications in hydrology and water studies by researchers not familiar with more complex statistical methods.

The objective of this study is then to develop a Mixed ensemble based system (MEBS) for Chl-a estimation in Quebec inland waters. The GQ application has permitted to develop an EBC composed of six classifiers and an EBE composed of twenty-one estimators. The MEBS has been evaluated by an adjusted k-fold cross-validation, confusion matrix, and the Coefficient of variance (CV).

\section{METHODOLOGICAL APPRAOCH}

\subsection{Mixed Ensemble Based System calibration}

The main key to enhance the AM performance is to avoid any mis-selection of the estimator. To handle this problem, it was necessary to determine an error variance on each AM thresholds $\left(\mathrm{V}_{1}\right.$ - and $\mathrm{V}_{2}$-threshold on Figure 1$)$. This was possible by means of statistical moments (mean $\left(\mu_{1}\right)$ and variance $\left.\left(\mu_{2}\right)\right)$ computation on the re-sampling vectors $\left(v_{1}\right.$ and $v_{2}$ ) corresponding to each of the AM thresholds. The generation of $v_{1}$ and $v_{2}$ was made by re-computing the $V_{1^{-}}$and $V_{2}$-threshold 25,000 times. Since the GQ requires the normality of the re-sampling vector, $v_{1}$ and $v_{2}$ were normalized using a boxcox transformation. Both vectors underwent a logarithmic transformation. The application of the GQ on $v_{1}$ and $v_{2}$ has allowed to compute an error margin (blue (upper-limit) and green (lower-limit) lines on the Figure 2) on either side of the two nominal thresholds (red lines on the Figure 2), which correspond to the MEBSEBC.
By partitioning the $\left[\mathrm{V}_{1}, \mathrm{~V}_{2}\right]$ feature space with the developed EBC, it was possible to calibrate the MEBS-EBE composed of twenty-one estimators. Thirteen possible scenarios for estimating the Chl-a concentration were identified (due to paging limit, this section is not detailed at the present paper). Each one comprises a set of estimators combined by means of their corresponding weighting coefficients $\mathrm{A} i$ and $\mathrm{A} j$ (Table 1), using Eq (1). The GQ allows also to compute the variance on Chl-a estimates using $\mathrm{Eq}(2)$.

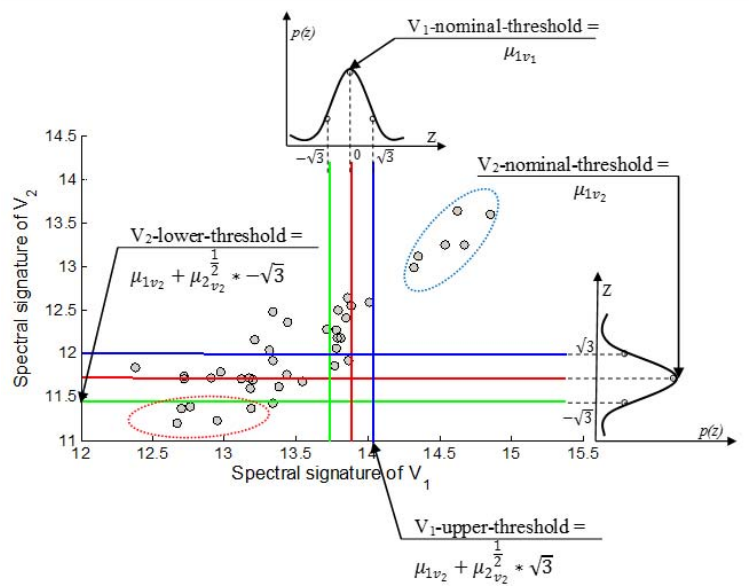

Figure 2. Scheme of the use of the Gaussian quadrature on 2dimensions using two variables $\left(\mathrm{V}_{1}\right.$ and $\left.\mathrm{V}_{2}\right)$ and its application to calibrate both classifiers and estimators of the mixed ensemble based system (MEBS). Red, blue and green lines represent, respectively, the nominal, upper and the lower thresholds of each variable (MEBS classifiers) and the sub-group of data surrounded by the blue ellipse represent the training data used to calibrate the estimator designed to estimate high chlorophyll-a concentrations whereas the sub-group of data surrounded by the red ellipse represent the training data used to calibrate the estimator designed to estimate low chlorophyll-a concentrations.

Table 1. Abscissas and weights for the standard normal distribution.

\begin{tabular}{c|c|c|}
\hline nodes & $z(i, j)$ & $A(i, j)$ \\
\hline 2 & $-\sqrt{\mathbf{3}}, \mathbf{0},+\sqrt{\mathbf{3}}$ & $\frac{\mathbf{1}}{\mathbf{6}}, \frac{\mathbf{2}}{\mathbf{3}}, \frac{\mathbf{1}}{\mathbf{6}}$ \\
{$[\mathrm{Chl}-\mathrm{a}]=\sum_{\mathrm{i}=0}^{\mathrm{n}} \mathrm{A}_{\mathrm{i}} \sum_{\mathrm{j}=0}^{\mathrm{n}} \mathrm{A}_{\mathrm{j}} *\left\{\mathrm{f}_{\mathrm{i}}(\right.$ Est $), \mathrm{f}_{\mathrm{j}}($ Est $\left.)\right\}$}
\end{tabular}

Where Est is estimator, $\mathrm{n}$ is the number of each involved Est, for each pixel, at the finale estimation and $A_{i}$ and $A_{j}$ are their corresponding weighting coefficients.

\subsection{Mixed ensemble based system validation}

To assess the performance of the MEBS, it was evaluated by $\mathrm{k}$-fold cross-validation, confusion matrix, and CV. The kfold cross-validation consists in temporary removing a bloc 
of samples from the dataset and to use the remaining samples as training data to estimate the removed samples with the pre-calibrated sub-model. Once all Chl-a measurements are estimated, the model performance can be evaluated using statistical indexes such as the coefficient of determination $\left(\mathrm{R}^{2}\right)$, relative bias (BIASr), relative root mean square error (RMSEr), and relative NASH-Sutcliffe efficiency $(\mathrm{Nr})$. Mathematical expressions of statistical indexes are as follow:

$$
\begin{aligned}
& \mathrm{R}^{2}=\left[\frac{\sum_{\mathrm{i}=1}^{\mathrm{n}}\left(\mathrm{M}_{\mathrm{i}}-\overline{\mathrm{M}}\right)(E s-\overline{\mathrm{Es}})}{\sqrt{\sum_{\mathrm{i}=1}^{\mathrm{n}}\left(\mathrm{M}_{\mathrm{i}}-\overline{\mathrm{M}}\right)^{2}} \sqrt{\sum_{\mathrm{i}=1}^{\mathrm{n}}\left(E s_{\mathrm{i}}-\overline{\mathrm{Es}}\right)^{2}}}\right]^{2} \\
& \text { BIASr }=\frac{1}{\mathrm{n}} \sum_{\mathrm{i}=1}^{\mathrm{n}}\left(\frac{E s_{i}-\mathrm{M}_{\mathrm{i}}}{\mathrm{M}_{\mathrm{i}}}\right) \\
& \text { RMSEr }=\sqrt{\frac{1}{\mathrm{n}} \sum_{\mathrm{i}=1}^{\mathrm{n}}\left(\frac{\left.E s_{i}-\mathrm{M}_{\mathrm{i}}\right)}{\mathrm{M}_{\mathrm{i}}}\right)^{2}} \\
& \mathrm{NASHr}=1-\frac{\sum_{\mathrm{i}=1}^{\mathrm{n}}\left(\frac{\mathrm{M}_{\mathrm{i}}-\mathrm{Es}}{\mathrm{M}_{\mathrm{i}}}\right)^{2}}{\sum_{\mathrm{i}=1}^{\mathrm{n}}\left(\frac{\mathrm{M}_{\mathrm{i}}-\overline{\mathrm{M}}}{\bar{M}}\right)^{2}}
\end{aligned}
$$

where $n$ is the training data size and $M, \bar{M}, E S$ and $\overline{E s}$ are, respectively, the measured and the estimated Chl-a concentrations with their corresponding averages.

A second data set, which is ordinal, was also used to assess the MEBS. This data set only indicates whether the cyanobacteria density was higher or lower than 20,000 cells per $\mathrm{mL}$, equivalent to $10 \mathrm{mg} \mathrm{Chl}-\mathrm{a} \mathrm{m}^{-3}$. Thus the evaluation time was made by confusion matrix.

A third evaluation by means of the Coefficient of variance (CV) was also made using Eq (7).

$\mathrm{CV}(\%)=\frac{\sqrt{\mu_{2}}}{\mu_{1}} * 100$

The CV measures the dispersion of individuals with respect to their mean. According to Martin and Gendron (2004), for a CV lower than $16 \%$ the mean can be considered as a reliable estimator, for a $\mathrm{CV}$ comprised between $16 \%$ and $33.3 \%$ the mean may comport some errors, and for a CV higher than $33.3 \%$ the dispersion is very important and the mean is no longer reliable [15].

\section{RESULTS AND DISCUSSION}

The k-fold cross-validation results were interesting as the coefficient of determination was about 0.98 showing that the MEBS can explain up to $98 \%$ of the variance of Chl-a concentration. The $\mathrm{Nr}$, which is a severe evaluation index, indicates that the MEBS is robust with a success rate of $95 \%$. The robustness of the model was also approved by the scatter-plot of in situ measurements versus their estimates (Figure 3) where all points are well distributed with respect to the line (1:1), confirming the accuracy of the MEBS estimations even at its extremities. The RMSEr was about $15 \%$ and the BIASr indicates that the MEBS underestimates Chl-a concentration by $2 \%$.

The confusion matrix results (Table 2) indicate that globally the MEBS performance was satisfactory, especially for estimations higher than $10 \mathrm{mg}$ Chl-a $\mathrm{m}^{-3}$ where the success rate reaches $96 \%$ and errors were lower than $8 \%$. However, the accuracy was relatively less good for concentrations inferior than $10 \mathrm{mg}$ Chl-a $\mathrm{m}^{-3}$ where omission and commission errors were about $9 \%$ and $19 \%$, respectively. Nevertheless, the overall performance of the MEBS, when compared to the AM, has increased from 0.87 to 0.92 and from 0.69 to 0.80 for the global success and Kappa-index, respectively [5].



Figure 3. Chlorophyll-a concentration estimated from the ensemble based model compared to in situ measurements.

Table 2. The MEBS confusion matrix results.

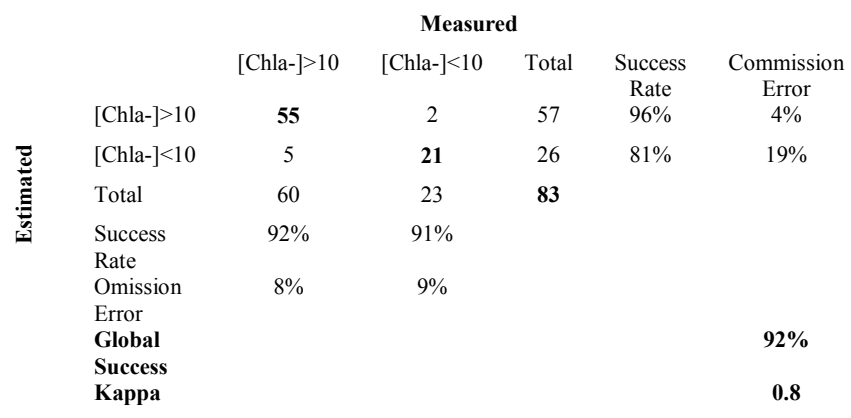

In order to qualitatively evaluate the performance of the MEBS modeling to the original model, both models were applied on a bloom detected at the Bay Missisquoi of Champlain Lake (Figure 4). A clear correspondence between the bloom shape drown by the MEBS (Figure 4.B) and the HAB extension detected on the MODIS true color image (green hue (Figure 4.A)) can be seen. This concordance is somehow lost for the AM, especially for 
low-to-moderate concentrations where the model seems to overestimates the Chl-a (Figure 4.C).

The Figure 5 represents the spatial distribution of $\mathrm{CV}$ computed over the bloom detected on the Figure 4. Substantially all of Chl-a estimates are reliable, especially in high blooming condition $(\mathrm{CVs} \approx 0)$. For the rest of the Missisquoi Bay (moderate-to-low conditions), the CVs are generally less than $10 \%$, except for some southern areas where it generally reaches $90 \%$. The $\mathrm{CV}$ results are quite obvious since the highest dispersion coincides with low Chla (southern areas of the Missisquoi Bay (Figure 4.B)) where the highest commission and commission errors were obtained (Table 3).
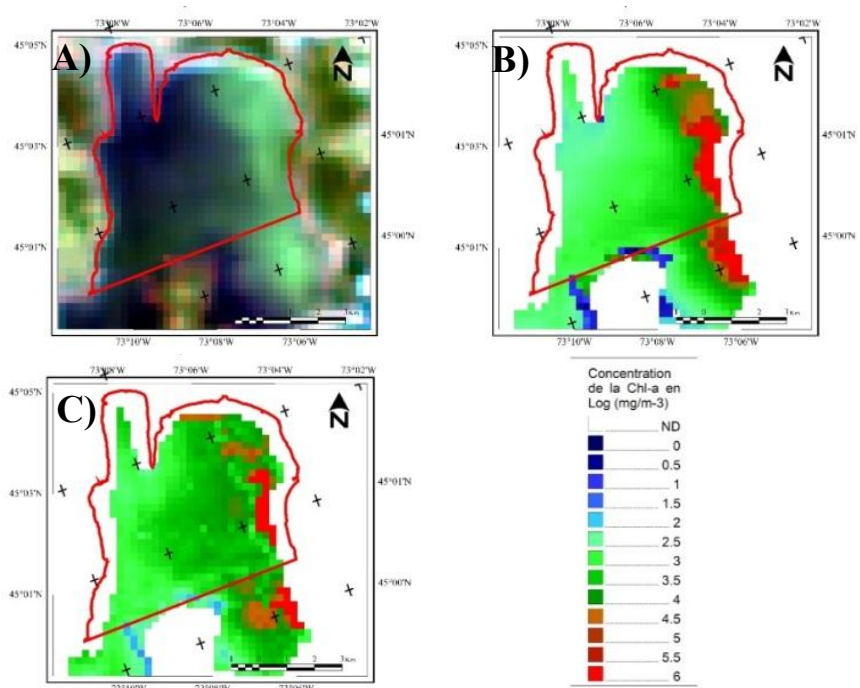

Figure 4. Comparison between the adaptive model and the mixed ensemble based system application on MODIS images. A) True color, B) OAM and C) AM. (19-Spet. 2001).

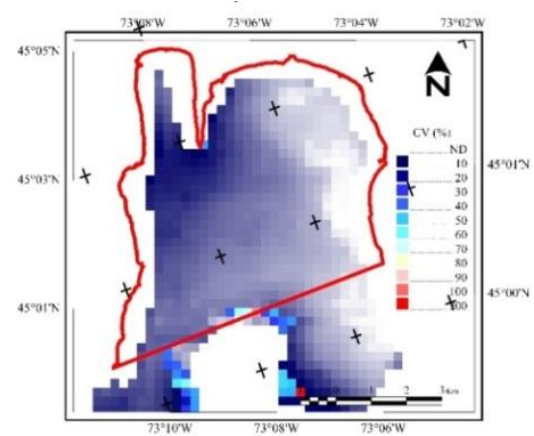

Figure 5. Spatial distribution of the coefficient of variance.

\section{CONCLUSION}

In general, when compared to the AM, the MEBS presented best results both quantitatively $\left(\mathrm{RMSE}=15 \%, \mathrm{R}^{2}=0.98\right.$, and Kappa-index $=0.80)$ and qualitatively $(\mathrm{CV}<10 \%)$ and showed a great potential to smooth the enormous contrast of Chl-a concentrations making the modelization more realistic, reliable, and accurate.

\section{REFERENCES}

[1] A. El-Alem, K. Chokmani, I. Laurion, and S. E. ElAdlouni, "Comparative Analysis of Four Models to Estimate Chlorophyll-a Concentration in Case-2 Waters Using MODerate Resolution Imaging Spectroradiometer (MODIS) Imagery," Remote Sensing, vol. 4, pp. 23732400, 2012.

[2] A. A. Gitelson, G. Dall'Olmo, W. Moses, D. C. Rundquist, T. Barrow, T. R. Fisher, et al., "A simple semi-analytical model for remote estimation of chlorophyll-a in turbid waters: Validation," Remote Sensing of Environment, vol. 112, pp. 3582-3593, 2008.

[3] C. Hu, "A novel ocean color index to detect floating algae in the global oceans," Remote Sensing of Environment, vol. 113, pp. 2118-2129, 10// 2009.

P. J. Baruah, M. Tamura, K. Oki, and H. Nishimura, "Neural network modeling of surface chlorophyll and sediment content in inland water from Landsat Thematic Mapper imagery using multidate spectrometer data," 2002, pp. 205-212.

A. El-Alem, K. Chokmani, and I. Laurion, "Apport de la télédétection spatiale dans le suivi des épisodes de fleur d'eau d'algues dans les lacs du Québec méridional," Institut National de la Recherche Scientifique, Centre Eau-Terre-Environnementjuin-2013 2013.

R.-H. Li and G. G. Belford, "Instability of decision tree classification algorithms," presented at the Proceedings of the eighth ACM SIGKDD international conference on Knowledge discovery and data mining, Edmonton, Alberta, Canada, 2002.

L. Rokach, "Ensemble-based classifiers," Artificial Intelligence Review, vol. 33, pp. 1-39, 2010/02/01 2010.

R. A. Jacobs, M. I. Jordan, S. J. Nowlan, and G. E. Hinton, "Adaptive mixtures of local experts," Neural Comput., vol. 3, pp. 79-87, 1991.

H. L. Cloke and F. Pappenberger, "Ensemble flood forecasting: A review," Journal of Hydrology, vol. 375, pp. 613-626, 2009.

C. Hung and J.-H. Chen, "A selective ensemble based on expected probabilities for bankruptcy prediction," Expert Systems with Applications, vol. 36, pp. 5297-5303, 2009.

R. Polikar, "Ensemble based systems in decision making," Circuits and Systems Magazine, IEEE, vol. 6, pp. 21-45, 2006.

L. Breiman, "Bagging Predictors," Machine Learning, vol. 24, pp. 123-140, 1996/08/01 1996.

K. S. Kelly and R. Krzysztofowicz, "A bivariate metaGaussian density for use in hydrology," Stochastic Hydrology and Hydraulics, vol. 11, pp. 17-31, 1997/02/01 1997.

H. Tørvi and T. Hertzberg, "Estimation of uncertainty in dynamic simulation results," Computers \& Chemical Engineering, vol. 21, Supplement, pp. S181-S185, 5/20/ 1997.

L. Martin and A. Gendron, Méthodes statistiques appliquées à la psychologie: traitement de données avec Excel, 2004. 